\title{
Armadura para columnas estructurales para un puente-grúa de 20 toneladas
}

Duvan Peña*

\section{Resumen}

Se realizó un diseño estructural de columnas para un puente-grúa con capacidad nominal de 20 toneladas. El diseño debe ajustarse a las dimensiones del lugar en el que se implementará. Inicialmente se propuso un diseño mecánico con base en la armadura tipo Warren, que se construye replicando en cada una de las caras de las columnas el patrón característico de la armadura Warren. De este diseño, se extrajo una armadura plana que se estudió a través del método de las secciones. Posteriormente, se realiza el análisis estático en tres dimensiones, usando el método de secciones y nodos, según sea necesario. Para este caso, se somete la estructura con una relación 2:1 sobre la carga nominal. A partir de análisis estructural, se determinaron los elementos bajo tensión y bajo compresión. Para este caso, los elementos diagonales experimentan tensión, mientras que los elementos verticales y horizontales están sometidos a compresión. También se demuestra que la armadura metálica es hiperestática o estáticamente indeterminada.

\section{Abstract}

A structural design of columns is made for a bridge crane with a nominal capacity of 20 tons. The design must follow the dimensions of the place to be implemented. Initially, a mechanical design is proposed based on the Warren type armor, which is built by replicating on each of the faces of the columns the characteristic pattern of Warren armor. From this design, a flat reinforcement was extracted to be studied, by means of method of sections. Subsequently, the static analysis is performed in three dimensions, using the method of sections and nodes as necessary. For this case, the structure is subjected with a 2: 1 ratio on the nominal load. From structural analysis, the elements under tension and under compression were determinated. On this case, the diagonal elements experienced tension, whereas the vertical and horizontal elements were subjected to compression. Also, the metallic armor is shown to be hyperstatic or statically indeterminate.
Cómo citar este artículo (APA): Peña, D. (2019). Armadura para Columnas Estructurales para un puente-grúa de 20 toneladas. Hashtag, 14, 39-49.

> Palabras clave: análisis estructural, armaduras metálicas, diseño mecánico, puente-grúa.

\section{$>$ Keywords:}

bridge-crane, mechanic design. metal armor, structural analysis. 


\section{Introducción}

$\mathrm{A}$ ctualmente, en la bodega 19, en el parque industrial Puerto Vallarta, se encuentra instalado un puente-grúa tipo pórtico, con capacidad nominal de 20 toneladas. Una de las características de este tipo de puente-grúas es que el movimiento longitudinal del pórtico se realiza a la altura del suelo. Sin embargo, esta característica ha causado incidentes de trabajo, como atrapamientos, choques y golpes. Por el momento, los incidentes no han comprometido la integridad de alguna persona. Se determinó que el nivel de riesgo es muy alto y requiere de medidas de control.

Según la norma GTC 45, existen dos tipos de controles: controles de ingeniería y controles al individuo (Instituto Colombiano de Normas Técnicas y Certificación, Icontec, 2010). Dentro de la matriz de riesgo se estableció utilizar una medida de control de ingeniería. Una solución es instalar vigas carrileras, con el fin de que la traslación del puente-grúa se realice a distinto nivel. Se busca, para las vigas carrileras, un diseño de armadura que permita controlar la condición insegura en la bodega, ocupar el mínimo espacio y soportar las cargas mecánicas que el puente-grúa trasmite durante el izado con carga nominal.

La instalación de vigas carrilleras elevadas permitirá controlar la condición insegura que representa un pórtico en movimiento, así como garantizar la capacidad operativa del puente-grúa. De esta forma, se permite la ejecución de las actividades laborales de la bodega de forma segura.El diseño estructural de las vigas carrileras integran todos los contenidos del análisis de estructuras aplicadas a armaduras y puntos de apoyo. Estos conceptos aplicados y desarrollados en el diseño y análisis de las vigas carrilleras son ampliamente usados en multitud de proyectos de ingeniería, es decir, los análisis inmersos en el diseño de este tipo de vigas, como casos de estudio, sientan la base para análisis estáticos de sistemas y máquinas de mayor complejidad estructural que un puente-grúa.

Dentro del diseño y análisis estructural, las armaduras se definen como la unión de varios elementos entre sí (Meriam y Kraige, 2012). La armadura es uno de los principales tipos de estructuras usadas en la ingeniería ya que proporcionan una solución práctica y económica para diseños que deben soportar las cargas que se le aplican con seguridad. Una armadura consta principalmente de elementos rectos que se conectan en nodos. Cada armadura está diseñada para soportar aquéllas cargas que actúan en su plano y por tanto pueden ser tratadas como estructuras bidimensionales. Para formar una armadura plana o bidimensional, se unen 2 o más armaduras triangulares. Así que, la figura geométrica base de una armadura plana es el triángulo. Cabe resaltar que las estructuras reales están hechas a partir de varias armaduras simples que forman una armadura espacial o tridimensional. La forma más simple de armadura espacial es un tetraedro (Beer, Johnston, Mazurek y Eisenberg, 2010).

Existen varias armaduras típicas que son ampliamente usadas para ofrecer soluciones estructurales. Destacan las armaduras tipo Pratt, tipo Howe, tipo Baltimore, entre otros. No obstante, la armadura tipo Warren destaca por su gran practicidad, ser económica y ofrecer buena resistencia mecánica (Hibbeler, 2012). Además, esta armadura permite la inclusión de barras montantes que dan rigidez a la estructura, lo que reduce la flexión de los elementos. Cabe señalar 
que en una armadura las barras están sometidas a dos tipos básicos de esfuerzos: la tensión y la comprensión. Es importante determinar las cargas mecánicas de cada elemento, así como si el elemento está sometido a tensión y a comprensión (Urban, 2008).

Figura 1. Armadura Warren sin y con barras montantes.

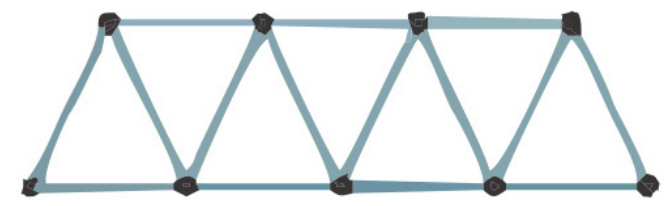

Armadura Warren sin barras montantes

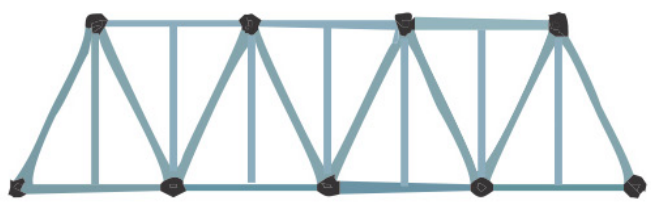

Armadura Warren con barras montante

Fuente: elaboración propia

Dentro del análisis de estructuras se utilizan técnicas para su análisis. Destacan el método de nodos y el método de secciones. El primer método se basa en que toda la armadura está en equilibrio, inclusive los nodos que forman la armadura (Meriam y Kraige, 2012). Para aplicar este método se debe trazar el diagrama de cuerpo libre de cada nodo y luego se aplican las ecuaciones de equilibrio de fuerzas para obtener las fuerzas que cada elemento aplica sobre el nodo. En cambio, el método de secciones busca extraer una parte de la armadura sobre la cual se encuentra las fuerzas que desea estudiarse, para después considerar las fuerzas de reacción. Este segundo método tiene la ventaja de usar la ecuación de momentos en equilibrio con el fin de despejar fuerzas desconocidas. Por último, se establecen las ecuaciones de equilibrio y se hallan las fuerzas desconocidas (Hibbeler, 2012). Estos dos métodos son la base del análisis estructural de armaduras planas y espaciales.

pasos. Primero, se debe considerar el sistema mecánico dentro de un punto de vista holístico y definir cuáles son los elementos estructurales a estudiar y diseñar, dentro del sistema mecánico considerado. Para facilitar este primer paso, se diseñó un diagrama de bloques, expuesto en la figura 2, que considera la trasmisión de las cargas de un elemento estructural a otro. A partir del diagrama de bloques se establecen las columnas estructurales como el objetivo del diseño. 
Figura 2. Diagrama de bloques de la trasmisión de las cargas de un elemento estructural a otro

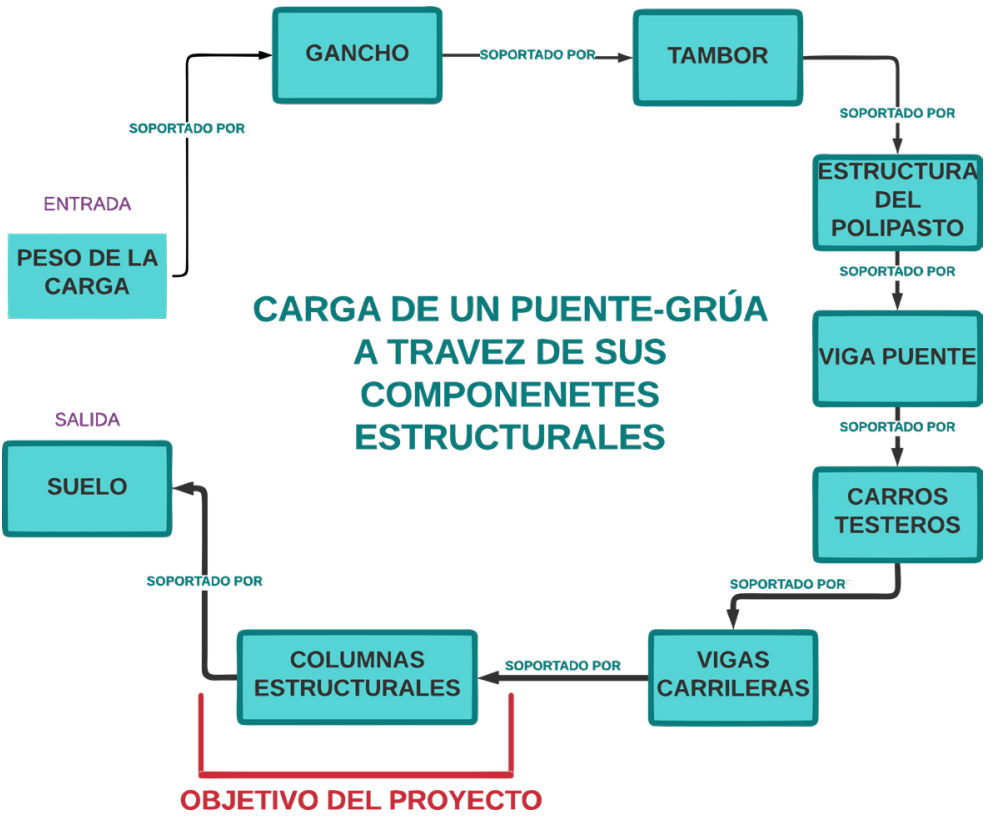

Fuente: elaboración propia.

Como segundo paso, se deben establecer los requerimientos funcionales del diseño estructural. Estos requerimientos se ajustan a las dimensiones espaciales del lugar donde se planea implementar el diseño, a las cargas mecánicas que van a ejercerse sobre la estructura, y al factor de seguridad calculado sobre la carga nominal del puente grúa. Se estiman las dimensiones a partir del puente grúa tipo pórtico, instalado actualmente, que son $7 \mathrm{~m}$ de altura, $40 \mathrm{~m}$ de longitud y $16 \mathrm{~m}$ de luz entre las vigas carrilleras. La carga nominal del puente grúa es de 20ton, y el factor de seguridad es de 2:1. Es decir, la carga nominal del puente grúa es de 20ton, pero la estructura debe soportar una carga distribuida de 40 ton. La figura 3 es un registro fotográfico actualizada de la bodega 19.

Figura 3. Registro fotográfico bodega 19

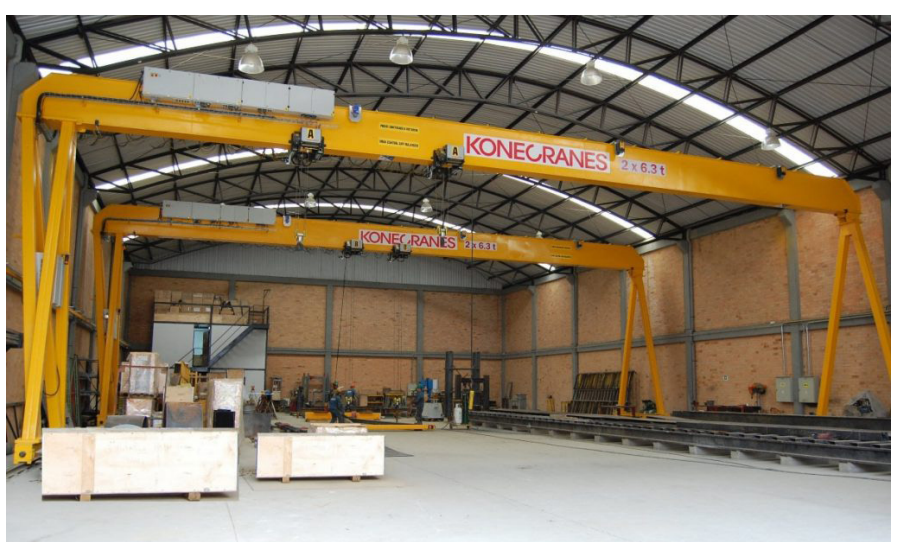

Fuente: Konecranes s.f. 
Una vez delimitado el objetivo del diseño y establecidos sus requerimientos del mismo, se procede a escoger una armadura típica, que servirá de base para el diseño. En este caso, se escoge la armadura tipo Warren porque permiten la inclusión de barras montantes que ayudan a rigidizar la estructura, y a disminuir la flexión, y es económica en relación a la cantidad de materiales (Urban, 2008). Luego, se establece un diseño estructural para las columnas estructurales. Posteriormente se aplica el análisis estático al diseño escogido. Se iniciará por un análisis bidimensional. Luego se realizará el análisis estático tridimensional. Dentro del análisis se consideran cuáles son los elementos sometidos a tensión y cuales a compresión. En la figura 4 se muestra la cadena de secuencia con los pasos que se siguen en la realización de este diseño mecánico.

Figura 4. Secuencia del diseño propuesto

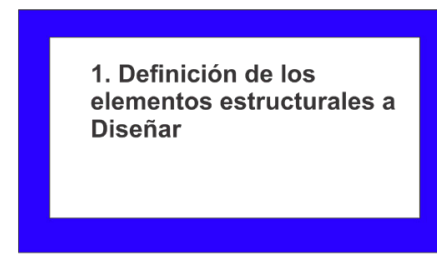

2. Definicion de los

Requerimientos del diseño:

Dimensiones

Carga Nominal

-Factor de seguridad

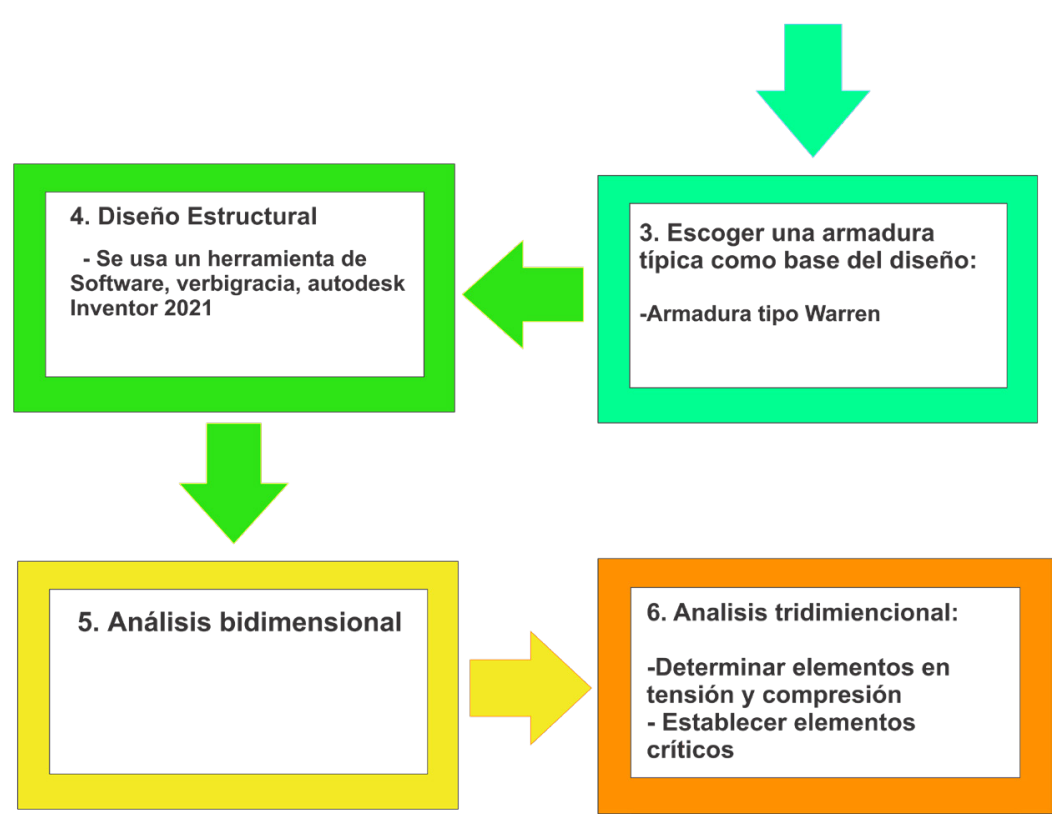

Fuente: elaboración propia.

\section{Resultados}

Inicialmente se desarrolló el siguiente diseño estructural para las columnas, con base en la armadura Warren con barras montantes:
Figura 5. Diseño 1 armadura Warren

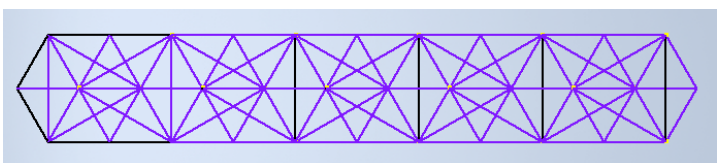


A partir de este diseño, se extrajo la cara frontal para realizar el análisis estático bidimensional. En la siguiente imagen se observa la cara frontal con cada uno de los nodos identificados y las dimensiones de la estructura:

Figura 6. Cara frontal.

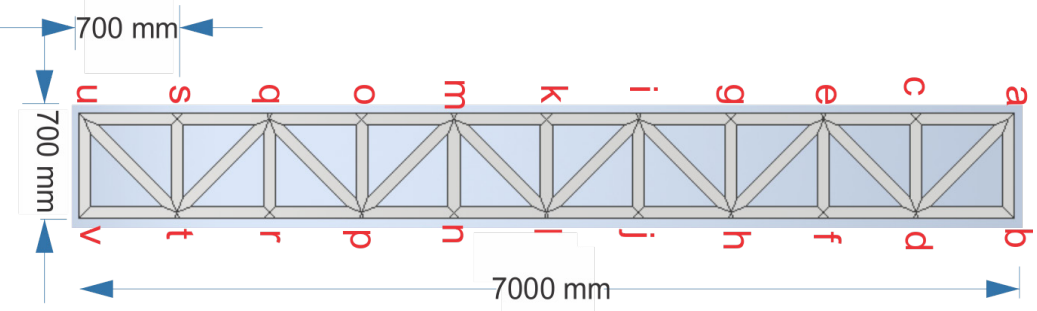

Fuente: elaboración propia

Con el fin de realizar el análisis estático bidimensional se definió la siguiente sección:

Figura 7. Sección cara frontal

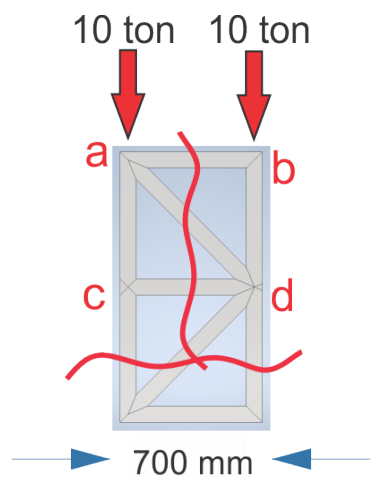

Fuente: elaboración propia.

Inicialmente se calculará la fuerza de reacción $\mathrm{C}_{\mathrm{y}}$ a partir de la sumatorias de momentos en el nodo d.

$$
\begin{gathered}
\sum M_{d}=10 \text { Ton } * g * 0.7 \mathrm{~m}-C_{y}=0 \\
C_{y}=10000 \mathrm{~kg} * 9.8 \mathrm{~m} / \mathrm{s}^{2} * 0.7 \mathrm{~m} \\
C_{y}=68.67 \mathrm{kN}
\end{gathered}
$$

Cabe resaltar que la fuerza $C_{y}$ equivale a la fuerza en el elemento CE y el elemento AC.

Enseguida se procede a realizar la sumatorias de fuerza en el eje Y para el nodo A y procedemos a calcular la fuerza sobre el elemento AD.

$$
\begin{gathered}
\sum F A_{y}=C_{y}+A D \cos 45^{\circ}-10 \text { Ton } * g=0 \\
A D \cos 45^{\circ}=10 \mathrm{Ton} * g-C_{y} \\
A D \cos 45^{\circ}=10000 \mathrm{~kg} * 9.8^{\mathrm{m}} / \mathrm{s}^{2}-68.67 \mathrm{kN} \\
A D=\frac{29.43 \mathrm{kN}}{\cos 45^{\circ}} \\
A D=41.62 \mathrm{kN}
\end{gathered}
$$

(Ecuación 2)

Después se hizo el cálculo de la fuerza de reacción $\mathrm{D}_{\mathrm{y}}$ a partir de la sumatorias de momentos en el nodo c.

$$
\begin{gathered}
\sum M_{c}=10 \text { Ton } * g * 0.7 \mathrm{~m}-D_{y}=0 \\
D_{y}=10000 \mathrm{~kg} * 9.8 \mathrm{~m} / \mathrm{s}^{2} * 0.7 \mathrm{~m} \\
D_{y}=68.67 \mathrm{kN} \\
\text { (Ecuación 3) }
\end{gathered}
$$


Cabe resaltar que la fuerza $\mathrm{D}_{\mathrm{y}}$ equivale a la fuerza en el elemento BD y el elemento DF.
Ahora se procede a realizar la sumatorias de fuerza en el eje $Y$ para el nodo D y procedemos a calcular la fuerza sobre el elemento DE

$$
\begin{gathered}
\sum F D_{y}=D_{y}+D E \cos 45^{\circ}-10 \text { Ton } * g-A D \cos 45^{\circ}=0 \\
D E \cos 45^{\circ}=10 \text { Ton } * g+A D \cos 45^{\circ}-D_{y} \\
D E \cos 45^{\circ}=10000 \mathrm{~kg} * 9.8^{\mathrm{o}} / \mathrm{s}^{2}+41.62 \mathrm{kN} \cos 45^{\circ}-68.67 \mathrm{kN} \\
D E=\frac{58.86 \mathrm{kN}}{\cos 45^{\circ}} \\
D E=83.24 \mathrm{kN}
\end{gathered}
$$

(Ecuación 4)

De la sección estudiada falta conocer la fuerza que somete al elemento AD y CD. Para hallar $\mathrm{AD}$, tenemos que:

$$
\begin{gathered}
\sum F A_{X}=A B-A D \cos 45^{\circ}=0 \\
A B=29.42 k N \\
\text { (Ecuación 5) }
\end{gathered}
$$

Para el caso de CD tenemos que:

$$
\begin{gathered}
\sum F D_{X}=-C D-A D \cos 45^{\circ}+D E \cos 45^{\circ}=0 \\
C D=58.85 k N-29.42 k N \\
C D=29.42 k N \\
\text { (Ecuación 6) }
\end{gathered}
$$

Se puede observar en las sumatorias de fuerzas realizadas sobre los nodos que los elementos verticales experimentan una fuerza en dirección hacia abajo, producida por la carga; y otra fuerza de dirección contraria, que comprime a los elementos verticales. Por lo tanto, los elementos verticales están trabajando en comprensión. Para el caso de los elementos horizontales sucede algo similar: las fuerza que soportan el elemento salen de los nodos estudiando hacia el elemento horizontal, lo que lo comprime. En contraste, los elementos diagonales experimentan fuerzas que van desde el elemento hacia el nodo, estirando al elemento. Por lo tanto, los elementos diagonales trabajan bajo tensión. La siguiente imagen resume cómo las fuerzas tensionan y comprimen los elementos de la armadura:

Figura 8. Fuerzas que actúan en la armadura

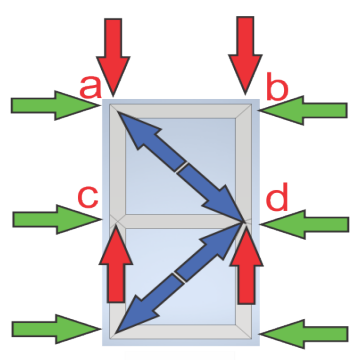

Fuente: elaboración propia.

Cabe señalar que como la estructura sigue un mismo patrón, las siguientes secciones están sometidas a las mismas fuerzas. En la siguiente imagen se observan los elementos de la armadura, así como los esfuerzos a los que son sometidos: 
Figura 9. Esfuerzos a los que se somete la armadura

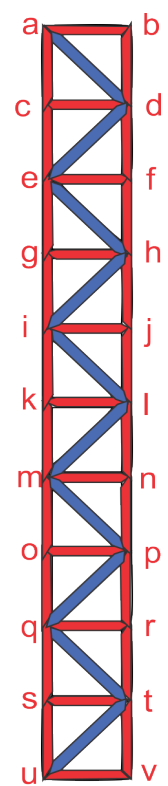

Fuente: elaboración propia.
En azul se representan los elementos a tensión y en rojo los a compresión. Los elementos horizontales experimentan una fuerza de $29.42 \mathrm{kN}$; los elementos verticales experimentan una fuerza de 68.67 kN. En cuanto a los elementos diagonales, todos están sometidos a una fuerza de 83.24 $\mathrm{kN}$, con la excepción del elemento AD que experimenta una tensión de $41.62 \mathrm{kN}$ y BD con una fuerza de $98.1 \mathrm{kN}$.

\section{Análisis tridimensional}

Para estudiar el análisis tridimensional de la armadura, se definió la siguiente sección, en la que se aplica una carga de 10 ton en los nodos A, B, C y D, según se muestra en la figura 10:

Figura 10. Análisis de esfuerzos 3D

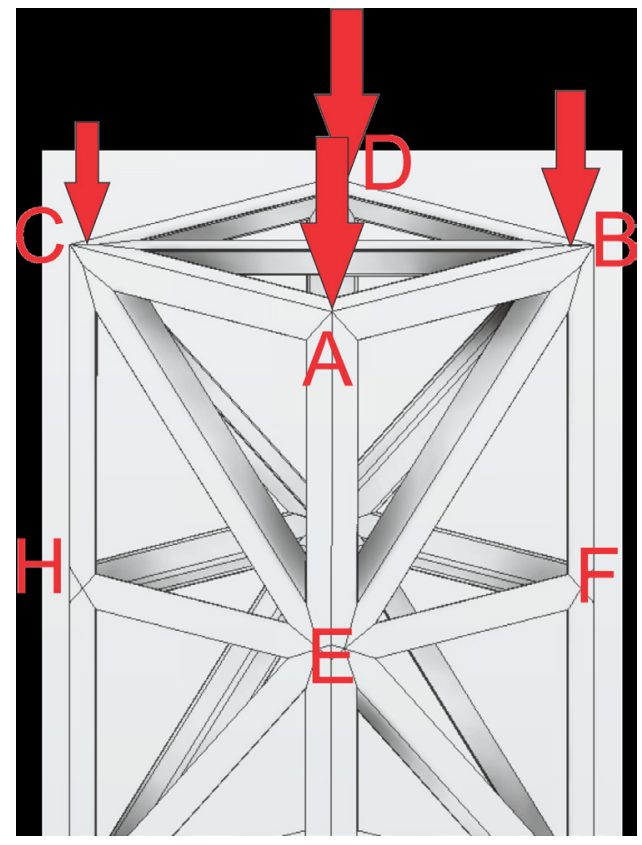

Fuente: elaboración propia. 
Iniciamos con la sumatoria de fuerzas en el eje $\mathrm{z}$ para el nodo A y D:

$$
\begin{gathered}
\sum F A_{z}=-98.1 k N+A_{z}=0 \\
\sum F D_{z}=-98.1 k N+D_{z}=0 \\
98.1 k N=D_{z}=A_{z}
\end{gathered}
$$

(Ecuación 7)

Se evidencia que las fuerzas $\mathrm{A}_{\mathrm{z}}$ y $\mathrm{D}_{\mathrm{z}}$ son de igual magnitud. Enseguida, se definen las sumatorias de momento para el nodo B, teniendo en cuenta que $C_{y}$ son las reacciones a la carga aplicada en ese nodo:

$$
\begin{gathered}
\sum M B=-0.7 j X(-98.1 k N K)+\left(-0.7 j X A_{z} K\right)+(-0.7 j+0.7 i) X(-98.1 k N K) \\
+(-0.7 j+0.7 i) X\left(K C_{z}\right)+0.7 i X(-98.1 k N K)+0.7 i X D_{z} K=0 \\
\sum M B=68.67 k N i-0.7 A_{z} i+68.67 k N i+68.67 k N j-0.7 C_{z} i-0.7 j C_{z} j \\
+68.67 k N j-0.7 D_{z} j=0 \\
\sum M B_{X}=68.67 k N i-0.7 A_{z} i+68.67 k N i-0.7 C_{z} i=0 \\
\sum M B_{y}=68.67 k N j-0.7 D_{z} j+68.67 k N j-0.7 C_{z} j=0
\end{gathered}
$$

(Ecuación 8)

$$
\begin{gathered}
0.7 D_{z}+0.7 C_{z}=137.34 k N \\
0.7 A_{z}+0.7 C_{z}=137.34 k N \\
D_{z}=A_{z} \\
C_{z}=D_{z}=A_{z}=98.1 \mathrm{kN}
\end{gathered}
$$

(Ecuación 9)

Las expresiones obtenidas de las sumatorias de momentos en el nodo B muestran que el comportamiento del momento en el eje $X$ y el eje $Y$ es igual. Ahora bien, si se tratara a las últimas dos expresiones algebraicas como un sistema de ecuaciones $2 \times 2$, se obtendría un sistema con infinitas soluciones, debido a que las dos expresiones son la misma recta en el plano cartesiano. Por lo tanto, el comportamiento estático es el mismo para el eje $\mathrm{X}$ y Y. También se evidencia que no existe momento con respecto al eje $\mathrm{Z}$.

Ahora se presenta el análisis de momentos en el nodo G. Este nodo es uno de los que más incógnitas tiene: 


$$
\begin{gathered}
\sum M G=-0.7 j X(-98.1 k N K)+\left(-0.7 j X H_{z} K\right)+(-0.7 j-0.7 i) X(-98.1 k N K) \\
+(-0.7 j-0.7 i) X\left(E_{z} K\right)-0.7 i X(-98.1 k N K)-0.7 i X F_{z} K=0 \\
\sum M G=68.67 k N i-0.7 H_{z} i+68.67 k N i-68.67 k N j-0.7 E_{z} i+0.7 j E_{z} j \\
-68.67 k N j+0.7 F_{z} j=0 \\
\sum M G_{X}=68.67 k N i-0.7 H_{z} i+68.67 k N i-0.7 E_{z} i=0 \\
\sum M G_{y}=-68.67 k N j+0.7 F_{z} j-68.67 k N j+0.7 E_{z} j=0 \\
0.7 H_{z}+0.7 E_{z}=137.34 k N \\
0.7 F_{z}+0.7 E_{z}=137.34 k N \\
C_{z}=D_{z}=A_{z}=98.1 k N
\end{gathered}
$$

(Ecuación 10)

De nuevo, las expresiones obtenidas de las sumatorias de momentos en el nodo $G$ muestran que el comportamiento del momento en el eje $X$ y el eje $\mathrm{Y}$ es igual. Ahora bien, si se tratara a las últimas dos expresiones algebraicas como un sistema de ecuaciones $2 \times 2$, se obtendría un sistema con infinitas soluciones, debido a que las dos expresiones son la misma recta en el plano cartesiano. Por lo tanto, el comportamiento estático es el mismo para el eje X y Y. Por otra parte, vuelve a evidenciarse que no existe momento con respecto al eje Z. Se continúa con la sumatorias de fuerzas para los nodos interconectados por medio de diagonales.

Iniciaremos con las sumatorias en el eje $\mathrm{Z}$ para los nodos B, C, E, G:

$$
\begin{aligned}
& \sum F B_{z}=-\cos 45^{\circ} G B-\cos 45^{\circ} G B+98.1 k N-B_{z}=0 \\
& \sum F C_{z}=-\cos 45^{\circ} G B-\cos 45^{\circ} G C+98.1 k N-C_{z}=0 \\
& \sum F E_{z}=\cos ^{\circ} 5^{\circ} G B+\cos 45^{\circ} G C+98.1 k N-E_{z}=0 \\
& \sum F G_{z}=\cos 45^{\circ} G B+\cos 45^{\circ} G C+98.1 k N-G_{z}=0
\end{aligned}
$$

(Ecuación 11)

A partir de estas expresiones, se puede observar que existen más incógnitas que ecuaciones. Por lo tanto, se puede definir a esta armadura espacial como estáticamente indeterminada o hiperestática. Entonces, no es posible continuar con el análisis estático de la armadura. 


\section{Conclusiones}

Se determinó que la armadura espacial es estáticamente indeterminada o hiperestática. No obstante, se realizaron algunas observaciones sobre la armadura. Los nodos con la igual cantidad de conexiones tienden a tener el mismo comportamiento estático, a saber, los nodos B y C, o los nodos E y G. Respecto de las sumatorias de fuerzas con respecto al eje $\mathrm{Z}$ se observa que en las diagonales la dirección de la fuerza se dirige hacia el nodo, es decir, que cuando la diagonal se conecta por debajo del nodo, la dirección de la fuerza jala el elemento hacia aquel. De esta forma, se determina que las diagonales trabajan en tensión.

En cuanto a los elementos verticales, se puede decir que están sometidos a una carga que coincide hacia $-\mathrm{K}$ y experimentan fuerzas de reacción que coinciden con $\mathrm{K}$. Por lo que las verticales están sometidas a compresión. Estas mismas observaciones se obtuvieron en el análisis en dos dimensiones de la armadura. A partir, del análisis en dos dimensiones se evidencia que los elementos horizontales también se encuentran bajo compresión.
Por otra parte, esta armadura ofrece algunas ventajas. Es ligera, gracias a que no es una columna maciza, en cuyo caso se usaría una en $\mathrm{L}$ para soportar las cargas. Además, la estructura es tetraédrica, lo que permite distribuir las cargas superiores. En el análisis bidimensional se observó que las cargas de todos los elementos, con excepción del elemento $\mathrm{BD}$, se encuentran por debajo de la carga aplicada de $98.1 \mathrm{kN}$ en los nodos superiores. Una posible desventaja es que hay uniones entre 9 elementos.

En principio se planteó la unión por soldadura. No obstante, las uniones en los nodos no son sencillas de realizar en la práctica, dado el número de elementos fijados en un solo nodo. Con el fin de continuar el estudio, se debe escoger una perfilaría, así como desarrollar los análisis relacionados a la resistencia del material. También es recomendable usar algún software de elementos finitos que permita simular el comportamiento de la armadura a diferentes cargas.

\section{Referencias}

Beer, F., Johnston, R., Mazurek, D. y Eisenberg, E. (2010). Mecánica Vectorial para Ingenieros: Estática. (9na ed.) Cuidad de México, México: McGraw Hill.

Hibbeler, R. (2012). Ingeniería Mecánica: Estática (12ma ed.). Cuidad de México, México: Pearson Education.

Instituto Colombiano de Normas Técnicas y Certificación, Icontec (2010). Guía Técnica Colombiana GTC45. Guía para la identificación de los peligros y la valoración de los riesgos en seguridad y salud ocupacional. Recuperado de https://n9.cl/m40dn

Konecranes Colombia S.A.S (s.f.). Gruas Konecranes. Recuperado de: https:/ /www.gruaskonecranes.com/nosotros/

Meriam, J., \& Kraige, L. (2012). Mecánica para Ingenieros Estática (7ma ed.) Virginia, Estados Unidos: Reverté

Urban, P. (2008). Construcción de Estructuras Metálicas. (4ta ed). Alicante, España: Club Universitario. 